\title{
GRAVITATIONAL LENSING AS A COSMOLOGICAL TOOL
}

\author{
JOACHIM WAMBSGANSS \\ Astrophysikalisches Institut Potsdam (Germany) \\ e-mail: jwambsganss@aip.de
}

AND

RENYUE CEN AND JEREMIAH P. OSTRIKER

Princeton University Observatory (USA)

e-mail: cen, jpo@astro.princeton.edu

\section{Results and Summary}

Gravitational lensing provides good test for cosmological models:

- directly measures mass density fluctuations to very distant objects

- no assumptions concerning bias needed

- Various cosmological models predict different matter distributions

- Here we investigate strong/weak lensing properties of cosmological models numerically [Wambsganss et al. ApJ (Feb. 1998)]

First results, strong lensing [Wambsganss et al. Science 268, 274 (1995)]:

- $\operatorname{SCDM}(\Omega=1)$ predicts far too many large separation lenses

- $\Lambda \mathrm{CDM}(\Omega=0.4, \Lambda=0.6)$ predicts few if any such lenses

First results, weak lensing [Wambsganss et al. ApJL 475, L81 (1997)]:

- "Standard candles" (i.e. SN Ia) get unavoidable "broadening" of luminosity function due to weak lensing by intervening matter

- Lensing induced dispersion for true standard candles in $\Lambda$ CDM universe is $0.04 \mathrm{mag}(0.02 \mathrm{mag})$ at redshift $z=1(z=0.5)$

Work in progress:

- Investigation of weak/strong lensing by clusters along line-of-sight

- Exploration of shear produced by large scale structure

- Lensing effects on microwave background 